\title{
Madhava de Sangamagrama
}

Mathématicien et astronome indien, Madhava de Sangamagrama est surtout connu pour ses travaux pionniers sur les développements en série entière des fonctions trigonométriques, ce que de nombreux analystes et historiens considèrent comme la transition entre les mathématiques antiques et les mathématiques modernes. Il a également donné ses lettres de noblesse à la grande école mathématique et astronomique du Kerala, dont le rayonnement scientifique lui a survécu pendant près de deux siècles.

\section{Riad HAIDAR, haidar@onera.fr}

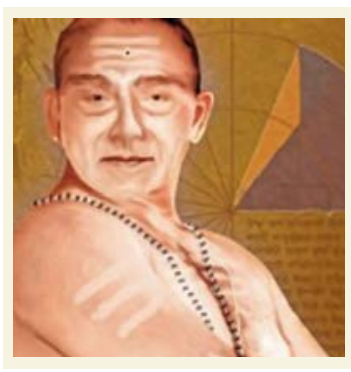

\begin{tabular}{l} 
EN BREF \\
$\begin{array}{l}\text { Environ } 1350 \text { - Naissance à Sangama- } \\
\text { gramma, près de Cochin (Inde) }\end{array}$ \\
$\begin{array}{l}\text { Développements en séries entières de sin, } \\
\text { arc-tan, etc. }\end{array}$ \\
\hline Formule pour l'estimation de $\pi$ \\
\hline 1425 - Décès à Sangamagramma \\
Portrait de Madhava de Sangamagrama
\end{tabular}

M adhava naît à Sangamagrama, un village près de Cochin, dans l'état de Kerala, sur la côte SudOuest de la péninsule Indienne (la fameuse côte de Malabar). Sangamagrama est composé des deux termes sangamam (union) et gramam (village). Il serait la traduction en sanskrit du mot dravidien Irinnatikutal, composé des termes iru (deux), annati (rivières) et kutal (union): on peut donc imaginer que le village a été fondé à la jonction de deux rivières à l'époque pré-médiévale. Aujourd'hui, ce lieu s'appelle Irinjalakuda, et comprend des parties des anciennes municipalités d'Irinjalakuda et d'Aloor. La famille de Madhava appartient à l'ethnie Namputiri, et l'histoire veut que le nom de la maison familiale, Irinna appilly, soit dû à une plante appelée iranni. Le nom de naissance de Madhava est donc Irinna appilly Madhavan.

\section{Tradition mathématique au Kerala}

Le Kerala hébergeait déjà une tradition mathématique très honorable avant l'époque de Madhava. Mais les historiens s'accordent à penser que cette tradition s'est affirmée et a pris un essor décisif avec les travaux fondateurs de Madhava. Il ne reste pratiquement aucune trace de son œuvre originale, mais elle est abondamment citée et référencée dans les œuvres des mathématiciens ultérieurs. En particulier le Tantrasangraha de l'astronome Nilakantha Somayaji, rédigé en sanskrit au tout début du $16^{\mathrm{e}}$ siècle, se réfere aux travaux de Madhava pour plusieurs développements en séries entières, dont ceux de sinus et arc-tangente. Et dans son grand-œuvre, le Yukti-Bhasa écrit en malayalam vers 1550 , Jyesthadeva donne les expressions des séries de Madhava sous forme de polynômes... Notons que le développement de la fonc-

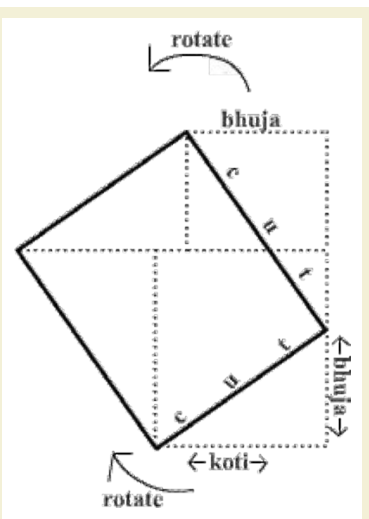

Illustration extraite du Yukti-Bhasa, sur le rôle du sinus.(C) Thunderboltz at English Wikipedia. tion arc-tangente sous la forme de $1 /\left(1+x^{2}\right)$ où $x=\tan \alpha$ a été retrouvé au $18^{\mathrm{e}}$ siècle par James Gregory en Écosse. Aussi cette série, qui a longtemps été appelée série de Gregory, a récemment été renommée série de Madhava-Gregory.

Au Kerala, Madhava initie une lignée d'astronomes et de mathématiciens féconds, que l'on peut tracer sur au moins deux siècles. Vatasseri Parameshvara Nambudiri, surtout connu pour avoir inventé une technique de calcul par récurrence du sinus, est un disciple direct. Puis, en succession, on peut citer Nilakantha Somayaji, Jyeshtadeva, Achyuta Pisharati of Trikkantiyur, et jusqu'à Melpathur Narayana Bhattathiri...

\section{Le saviez-vous?}

Les mathématiques indiennes sont d'une tradition abstraite et peu orientée vers la pratique, à quelques exceptions notables. Ainsi, c'est avec les grands astronomes tels que Vararuci ( $4^{\mathrm{e}}$ siècle), Âryabhata ( $5^{\mathrm{e}}$ siècle), ou encore Sankaranarayana ( $9^{\text {e }}$ siècle), qu'elles nouent un lien fécond avec l'astronomie. Dans son œuvre majeure I'Āryabhaìya, traité d'astronomie écrit en vers aux alentours de 499, Âryabhata présente ses théories astronomiques et mathématiques selon lesquelles la Terre tourne autour de son axe, les distances galactiques sont exprimées par rapport à la distance Terre-Soleil, et les planètes obéissent à un système héliocentrique et suivent des orbites elliptiques. Il suggère que la lumière de la Lune est en fait celle du Soleil réfléchie par notre satellite. Il explique correctement les éclipses du Soleil et de la Lune, alors que I'hindouisme attribue ces phénomènes au démon Râhu. Il estime la durée d'une année à 365 jours 6 heures 12 minutes et 30 secondes, une valeur précise à quelques minutes près... La plupart de ces avancées seront reprises et prolongées par les mathématiciens et astronomes de l'école du Kerala, probablement sous l'impulsion créatrice de Madhava. 


\section{Le calcull de $\pi$}

À partir de la série de Madhava-Gregory, évaluée pour $x=1$, on retrouve la formule de Madhava-Leibniz qui fournit une estimation du nombre $\pi$. Cette formule, dont la version originale est perdue, est rappelée dans le Mahajyanayana prakara (littéralement, méthodes des sinus), un ouvrage du $16^{\mathrm{e}}$ siècle qui se réfere au travail de Madhava:

$$
\sum_{\mathrm{n}=0}^{\infty} \frac{(-1)^{\mathrm{n}}}{2 n+1}=\frac{1}{1}-\frac{1}{3}+\frac{1}{5}-\frac{1}{7}+\frac{1}{9}-\cdots=\frac{\pi}{4}
$$

De façon remarquable, on y trouve également un terme de correction, qui permet d'estimer la précision de la somme sur $n$ termes de la série. Madhava en a établi trois formes, dont la plus raffinée s'écrit: $R n=\left(n^{2}+1\right) /\left(4 n^{3}+5 n\right)$, permettant d'atteindre une très grande précision sur l'estimation de $\pi$.

\section{Astronomie}

En héritier d'Âryabhata, Madhva s'intéresse aux mouvements des astres, et leur applique ses découvertes mathématiques. Il établit une procédure pour déterminer les positions de la Lune avec une périodicité de 36 minutes, et bâtit des méthodes pour estimer les mouvements des planètes. Dans le Yukti-Bhasa, Jyesthadeva évoque une technique de calcul appelée sankalitam (littéralement, collection ou ramassage), également attribuée à Madhava et qui correspond, en langage moderne, à l'intégration. On peut y lire ekadyekothara pada sankalitam samam padavargatbinte pakuti, ce qui signifie: 1 'intégration d'une variable (pada) est égale à la moitié de cette variable élevée au carré (varga $)^{1}$. Dans le même ouvrage, un résultat lié au sankalitam indique que l'aire sous une courbe correspond à son intégrale.

\section{Un liein avec l'Europe?}

Aux $15^{\mathrm{e}}$ et $16^{\mathrm{e}}$ siècles, le port de Muziris, près de Sangamagrama, est un centre important pour le commerce maritime alors en plein essor sur la côte de Malabar, et un certain nombre de missionnaires et de commerçants jésuites y sont actifs. Bien qu'aucune traduction des textes indiens (i.e., en sanskrit ou en malayalam) n'ait pour le moment été retrouvée, quelques historiens soutiennent la thèse selon laquelle cette proximité, ainsi que la renommée de l'école du Kerala, ont pu favoriser le transfert d'idées vers l'Europe, et donc avoir une influence sur les développements européens dans l'analyse et le calcul..

\section{RÉFÉRENCES}

[1] K.V. Sharma, S. Hariharan, Yukti-Bhasa of Jyesthadeva - A book on rationales in Indian Mathematics and Astronomy, Indian Journal of History of Science (1990).

[2] J.J. O'Connor, E.F. Robertson, Madhava of Sangamagramma, MacTutor History of Mathematics (2000).

On peut aussi le lire ainsi : I'intégrale de $x \mathrm{~d} x$ est égale à $x^{2} / 2$.

\section{acal $\left.\right|^{\text {bi }}$}

\section{Solutions Photoniques d'Acal BFi}

Acal BFi (anciennement BFi Optilas) est un acteur majeur en photonique depuis 1973 et offre une gamme complète de produits et services pour l'industrie de la Photonique. De la génération de la lumière (lasers, diodes, LEDs) et sa mise en forme (composants optiques, scanners/galvanomètres et modulateurs) jusqu'à sa détection (d'une simple photodiode aux systèmes spectroscopiques ou photométriques sophistiqués), le photon est le coeur de notre expertise.

Grâce à ses partenaires et à ses capacités de conception interne, Acal BFia lancé des solutions novatrices sur ce marché en pleine croissance. L'objectifétait de mettre à disposition des produits faciles d'utilisation à un prix compétitif, sans jamais compromettre la qualité. Parmi nos solutions les plus prisées, citons:

- Solutions lasers pulsés et continus toutes gammes de puissance et protections associées,

Solutions optiques standards et sur cahier des charges,

- Solutions de caractérisation goniométrique des LED et luminaires,

Solutions abordables pour les mesures de lumière et de couleur,

Colorimètres d'imagerie 2D haute qualité à des prix attractifs, Solutions ultra-efficaces de mise en forme de faisceau pour les LED et sources de lumière,

- Solutions novatrice de densification énergétique optique des LED (ex: $100 \mathrm{~W} / \mathrm{cm}^{2}$ à $365 \mathrm{~nm}$ ).

\section{Exemple de conception interne}

Pour répondre à la demande des clients d'Acal BFi recherchant une solution facile à utiliser etéconomique, nos ingénieurs ont conçu un nouveau spectroradiomètre/spectrocolorimètre. Cet appareil super-compact est alimenté sur batterie et intègre un pointeur laser, ainsi qu'un obturateur mécanique. Il offre des niveaux élevés de résolution, de précision et de sensibilité, avec un temps d' intégration court. Très simpleà utiliser, il est contrôlé avec un écran tactile et/ou des interfaces USB 2.0 / Wi-Fi. Les données peuvent être facilement exportées avec une clé USB. Cet outil est idéal pour un grand nombre d'applications, notamment:Projecteurs lasers, caractérisation LED/OLED/Ecran, automobile et ferroviaire, avionique, affichage tête haute, éclairage, cinéma et post-production.

\section{CONTACT}

\section{Acal BFi}

Département Photonique Tél. : 01607959 06/38 wuw.acalbfi.fr 